doi:10.17659/01.2020.0070

Journal of Case Reports 2020;10(4):261-265

\title{
Infected Diaphyseal Non-union of Radius Managed with Fibular Graft and Intramedullary Fixation: Case Report on Simple Technique for Complex Case
}

\author{
Lavindra Tomar, Gaurav Govil, Pawan Dhawan \\ Department of Orthopaedics, Max Super Speciality Hospital, 108 A, I.P. Extension, Patparganj, Delhi - 110092, India.
}

Corresponding Author:

Dr. Gaurav Govil

Email: gauravgovil@yahoo.co.in

This is an Open Access article distributed under the terms of the Creative Commons Attribution License (creativecommons.org/ licenses/by/3.0).

Received : July 7, 2020

Accepted : October 27, 2020

Published : December 25, 2020

\begin{abstract}
Background: Management of infected gap non-union of forearm bones is a challenging task. Various methods of treatment available in the literature are cortical tibial graft, ulnar segment graft, iliac crest graft, cancellous insert graft, vascularized fibular graft, bone transport by ring fixator and Nicoll's method with plating of iliac crest graft. Case Report: We report a complex case of infected non-union of radius managed with simple reconstruction of gap defect with a free non-vascularised fibular graft and supplemented by cortico-cancellous autologous bone graft. An intra-medullary wire was preferred over plating for fixation. At 18 months follow up, the patient is symptom free and is able to carry out the daily activities with ease. Conclusion: The procedure is economically viable and easily reproducible.
\end{abstract}

Keywords: Bone Fractures, Bone Transplantation, Bone Wires, Fibula, Forearm.

\section{Introduction}

Emphasis on anatomic reduction of forearm fractures and strict adherence to stable fixation techniques have reduced the rate of non-unions in the forearm to less than 3\% [1]. Open injuries, highly comminuted fractures, significant soft-tissue injury, inadequate surgical fixation, and infection are known predisposing factors to forearm nonunions [1,2]. The infected non-unions in the forearm are rare and the problem is complex due to the presence of bone necrosis, segmental bone loss, sinus tract formation, fracture instability, and scar adhesion of the soft tissues [2,3]. A large diaphyseal, segmental bone defect is a challenging problem in orthopaedic practice. Very few reports in the literature are available deliberating on the treatment strategies and functional outcomes $[1,2]$. Procedures used in the reconstruction of segmental defects of both bones of the forearm should, whenever possible, preserve forearm pronation and supination.

\section{Case Report}

A nineteen-year-old male patient presented to our department with post-traumatic infected gap nonunion of middle two third and lower one third region left radius with a discharging sinus. He was previously operated elsewhere and was found to have undergone repeated surgical interventions.

There was history of fall at home four months back sustaining a middle third and lower third junction forearm fracture of radius and ulna at the same level [Fig.1a,b]. Surgical stabilization by narrow 3.5 DCP plate was done for both fractures at a health care facility elsewhere on second day of injury [Fig.1c,d]. Infection and discharge from radial end ensued within three weeks of primary surgery. Antibiotic and regular dressings were unable to control discharge. Persistence of discharge followed for two months. Secondary procedure of radial plate removal, debridement and JESS distractor application was done 
[Fig.2a,b]. Radiograph revealed sequestration of radial segment under the plate with lysis along screw holes with periosteal reaction [Fig.2c,d]. Redo debridement under antibiotic coverage along with removal of sequestrum from radius followed creating a radial gap defect of around $7 \mathrm{~cm}$ [Fig. 2e,f]. It was followed by six weeks of appropriate antibiotic therapy.

Patient presented to us after four months of his index surgery with persistence of low-grade infection with a discharging sinus and compounded by tissue cicatrisation, joint contractures of thumb and wrist with muscle wasting. The sinus was situated in the centre of the previous surgical wound. Radiograph revealed loss of radial segment of approximately $7 \mathrm{~cm}$ length, JESS distractor in radius and ulnar plate in situ with loss of radial dominance at wrist. At the time of presentation, he was found to have sterile culture reports, normal leucocyte count with elevated levels of ESR and CRP. Range of elbow movements was full. Forearm movements were painful allowing only $20^{\circ}$ pronation. Evidence of low-grade pin tract infection was present.

After initial management with debridement and antibiotics for 3 weeks, repeat ESR and CRP were normal. Indigenous method consisting of ipsilateral free non-vascularized fibular graft with a Kirshner wire fixation through the graft to restore the length of radius was done [Fig.3a,b]. Under general anaesthesia, the forearm external fixator was removed first. The surgical wound track which previously got infected was then cleared with thorough debridement until the graft bed appeared red and bled freely, along with appropriate antibiotic. The proximal and distal stump ends were cut to create raw fresh vascularized bony ends. Both ends were drilled retrograde with 2.7 $\mathrm{mm}$ drill bit to open medullary cavity. The bone gap was measured intra-operatively to $10 \mathrm{~cm}$. Now the ipsilateral fibular graft of approximately $12 \mathrm{~cm}$ was harvested. The block of graft taken was trimmed at both ends to have long block of graft of desired

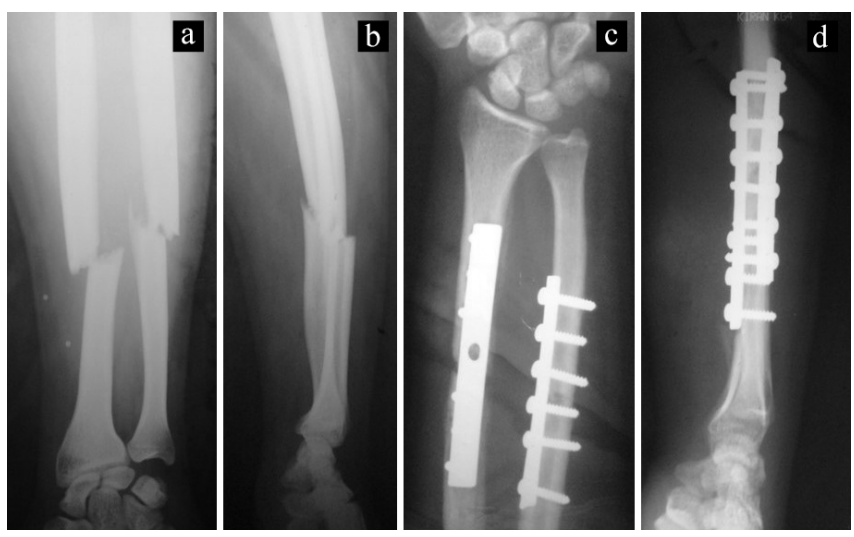

Fig.1:(a,b): Immediate post-trauma radiograph with AP and lateral view showing radius ulna fracture; (c,d): Radiograph after fixation by DCP plate.

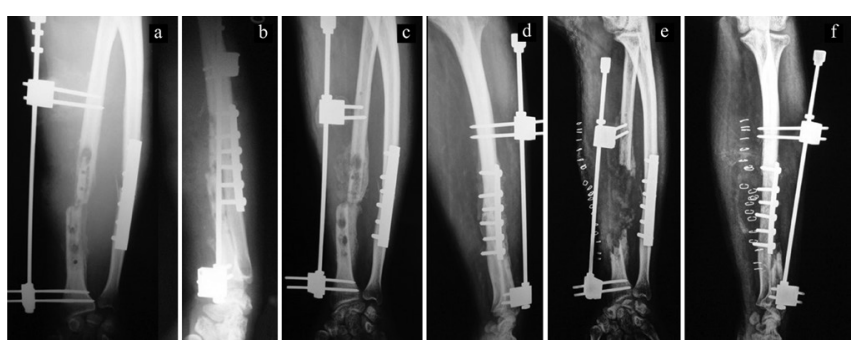

Fig.2:(a,b): Radiograph with AP and lateral views after radial plate removed and JESS fixator applied at two months post primary surgery; $\mathbf{( c , d ) : ~ I n t e r m e d i a t e ~ r a d i o g r a p h s ~}$ showed periosteal reaction along proximal and distal fracture ends with lysis; (e,f): Radiograph after removal of sequestered radial fragment with gap defect of around $7 \mathrm{~cm}$.

length. The block was then reamed eccentrically with a $2 \mathrm{~mm}$ Kirshner wire through and through its longitudinal axis. A smooth K-wire of determined length was passed into the distal fragment of radius and as the wire protruded out through the distal stump the fibular graft was threaded on to the wire. As soon as it protruded out through the other end of the graft, the proximal fragment reduced over the wire with angulation and distraction techniques. Ipsilateral autologous cancellous bone chips were added to both fracture ends. Haemostasis was achieved and all wound were closed. Postoperatively above elbow slab was given for a period of two weeks with conversion to cast for another four weeks. The patient was encouraged active finger movements and was serially followed 

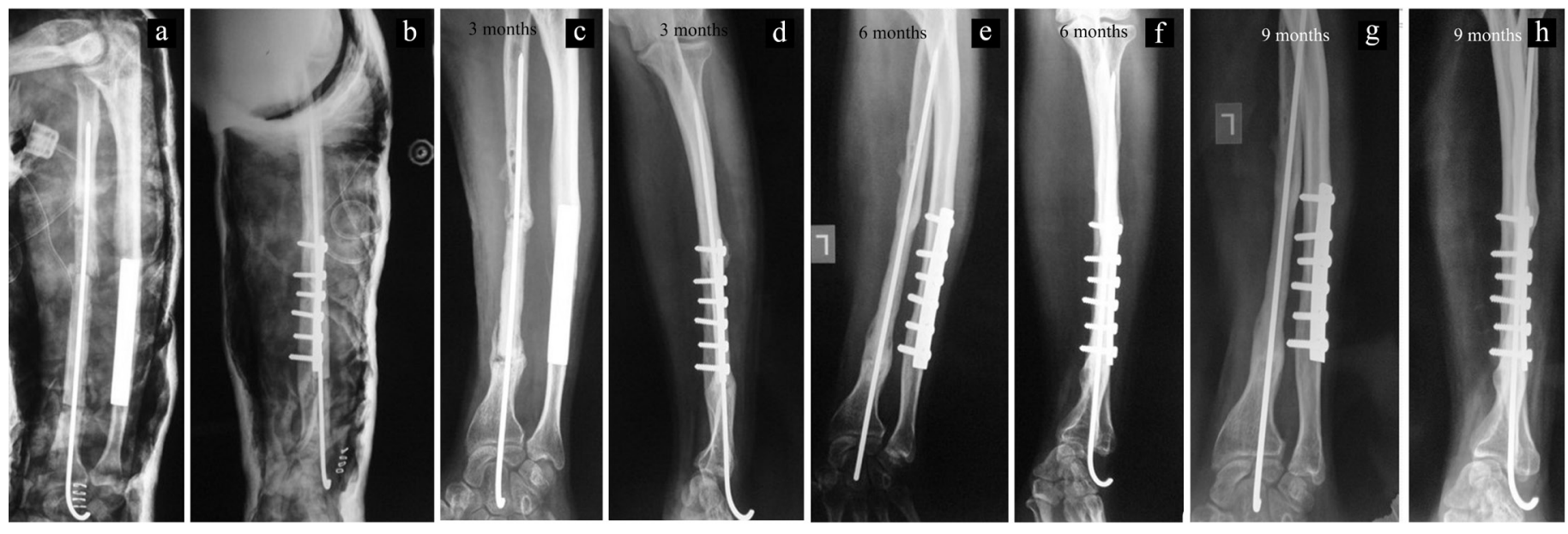

Fig.3:(a,b): Radiograph with AP and lateral views of immediate post-operative period with non-vascularized fibular strut graft and intramedullary fixation; (c,d): At three months, showed bridging callus; (e,f): At six months, good consolidation; (g,h): At nine months, graft incorporated and healed.

up with radiographs at three months interval [Fig.3c,d] showed bridging callus, at six months interval [Fig.3e,f] showed consolidation. At the end of nine months post operatively evidence of union was found at both ends of the graft [Fig.3g,h] with loss of radial dominance. At twenty months post-operative status patient regained satisfactory forearm and hand functions for his daily activities [Fig.4a-f]. There was no graft fracture. The patient was offered corrective procedure with ulna shortening, step cut osteotomy and re-plating, to restore the radial dominance. However, he did not consent for further intervention as his daily activities were painless and comfortable.

\section{Discussion}

Non-union can be caused by factors such as comminution or segmental fractures, bone defects, interposition at the fracture site, disruption of the blood supply in the fracture fragments, inadequate stabilization and local infection [1,4]. Problems involved in bridging or regenerating areas of skeletal loss with viable bone while maintaining limb length and alignment, along with satisfactory function remains a substantial challenge [5]. The options for defect management include autogenous cancellous bone graft, autogenous cortico-
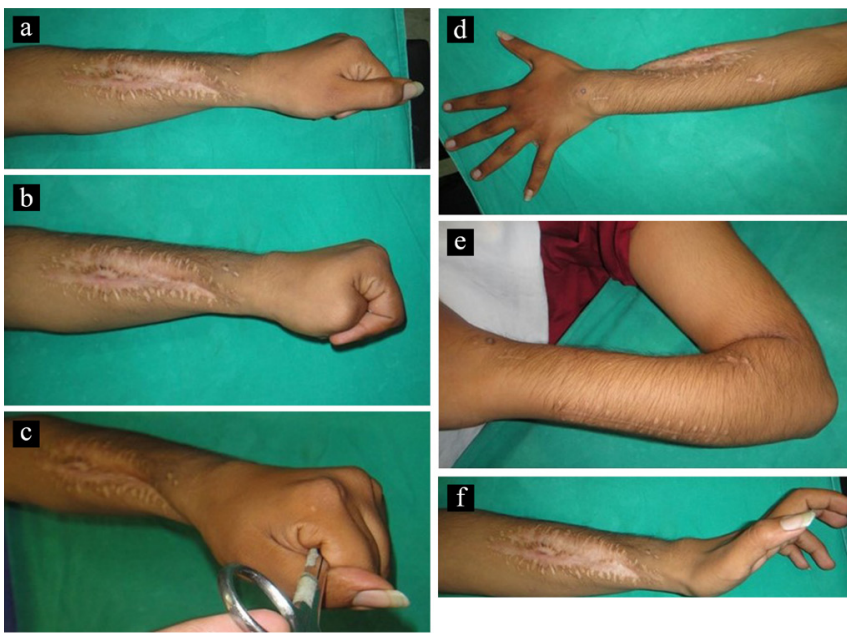

Fig.4: At twenty months follow up, good function and grip with healed scars.

cancellous bone graft and vascularized bone grafts (typically for the radius) in atrophic non-union [1]. Surgical treatment aims to regain proper length, restore the anatomy and recover function of the forearm $[2,4,5,6]$.

Non vascularized fibular graft is used to manage a diaphyseal forearm gap defect. It is a long, tubular, strong autogenous bone graft readily available [7]. Sacrifice of the fibula does not appear to have any detectable functional disadvantage and 
donor site morbidity [7-9]. Clinical studies have suggested that the distal six $\mathrm{cm}$ of fibula should be preserved to maintain lateral stability of the ankle $[3,5.7]$. The various studies found no correlation between the length of the non-vascularized fibular graft and the rate of healing.

Success of operative procedures involving non-vascularised autogenous bone graft depends on the ability of the surrounding soft tissues to withstand the operative manipulation and to revascularise the transplanted bone graft [8]. Despite a high success rate, the danger of resorption, a high incidence of stress fractures, and the lack of biological activity have been thought to be disadvantages of non-vascularized fibular grafts compared to vascularized grafts $[1,7,9]$. Though vascularised grafts are significantly stronger than conventional fibular grafts however, graft fracture are known to occur in both $[5,8]$. In addition, donor site morbidity following graft or flap procedures should also be considered.

Illizarov bone transport procedures have provided alternatives for the reconstruction of composite skeletal and soft-tissue defects; however, such procedures are complex and lengthy and have been associated with many complications $[7,8]$. We achieved defect filling with osteo-inductive cortico-cancellous iliac crest graft and osteoconductive fibular graft block and internal fixation by nail imparting stability to the fracture. In our procedure removal of nail does not require reexposure of graft area. We used less metallic implant material which is advantageous over plating. The procedure allowed early rehabilitation. Nailing proved a useful option in our patient. We agree that strong and stable fixation is must for achieving union and locking plates are more preferred over conventional non-locking plates with bi-cortical locked screw purchase though their superiority remains questionable [1,7]. Adjacent soft tissue viability and potential for the coverage of implant by native tissues are considerations in choosing an implant. The patient was apprehensive after failure with a plate and it challenged the decision making for implant selection.

One of the important factors in achieving functional success is the restoration of radial bowing [4]. Using a free non-vascularised fibular graft, excellent results in two cases of segmental ulnar defect with an average length of $9 \mathrm{~cm}$ were achieved [8]. The Nicoll's technique of cortical iliac graft with plating is recommended only in the absence of infection and if the bony gap does not exceed $50 \mathrm{~mm}$ [4]. We modified Nicoll's technique by using a flexible nail for fixing the graft. Also, we applied this method for a bone defect of $10 \mathrm{~cm}$ which is more than the proposed upper limit of fixation.

There are few reports to suggest superiority of any treatment options advocated. Successful outcome makes this a useful alternative in treatment of infective non-unions. We comprehend that significant challenges may arise with graft collapse, resorption and failure necessitating considerations of other treatment modalities.

\section{Conclusion}

Surgeons should opt for a treatment modality in an infected diaphyseal gap non-union of forearm considering challenges in securing a healthy surgical bed for reconstruction, selection of graft options for defect management and use of implant for secure fixation. The asepsis protocol should be adhered to in a primary surgery. We require simplifying the complex problem, and making it economically viable and easily reproducible.

Contributors: LT: conceptualization, patient management; GG: data collection, manuscript writing, patient management; PD: manuscript editing. GG will act as a study guarantor. All authors approved the final version of this manuscript.

Funding: None; Competing interests: None stated.

\section{References}

1. Kloen P, Buijze GA, Ring D. Management of forearm nonunions: current concepts. Strat Traum Limb Recon. 2012;7:1-11. 
2. Prasarn ML, Ouellette EA, Miller DR. Infected nonunions of diaphyseal fractures of the forearm. Arch Orthop Trauma Surg. 2010;130:867-873.

3. Nazim M, Nizamuddin MK. Fibula bone grafting in infected gap non-union: A prospective case series. National Journal of Clinical Orthopaedics. 2019;3(1):610.

4. Venkateswarlu BSS, Manikumar CJ. Infected gap nonunion of radius treated with modified Nicoll's technique: A case report. Journal of Clinical and Diagnostic Research. 2015;9(2):RD01-RD02.

5. Shah KB, Kamath J, Jayasheelan N, Danda R, Rai MK. Non-vascularized fibular strut grafting in management of bone defects. Online J Health Allied Scs. 2015;14(4):14.
6. Parihar M, Ahuja D. Infected Nonunion of radius and ulna - Strategy of approach. Journal of Orthopaedic Case Reports. 2012;2(4):26-31.

7. Nataraj B, Singh V, Pathak AC, Jain M, Khapane V. Nonvascularized fibula and corticocancellous bone grafting for gap nonunion of lower limb-retrospective study of 18 cases an age-old technique revisited. Eur Orthop Traumatol. 2014;5:277-283.

8. El-Sayed M, El-Hadidi M, El-Adl W. Free nonvascularised fibular graft for treatment of post-traumatic bone defects. Acta Orthop. Belg. 2007;73:70-76.

9. Mahdi M, Hafidh A. Free non-vascularized fibular graft in treatment of bone defects. Bas J Surg. 2007;13:69-75. 